\title{
Minimum energy control of fractional positive electrical circuits
}

\author{
TADEUSZ KACZOREK
}

\author{
Faculty of Electrical Engineering \\ University of Technology \\ Wiejska 45D, 15-351 Bialystok \\ e-mail:kaczorek@isep.pw.edu.pl
}

(Received: 26.12.2014, revised: 17.08.2015)

\begin{abstract}
Minimum energy control problem for the fractional positive electrical circuits is formulated and solved. Sufficient conditions for the existence of solution to the problem are established. A procedure for solving of the problem is proposed and illustrated by an example of fractional positive electrical circuit.

Key words: fractional, positive, electrical circuits, minimum energy control, bounded inputs, procedure
\end{abstract}

\section{Introduction}

A dynamical system is called positive if its trajectory starting from any nonnegative initial state remains forever in the positive orthant for all nonnegative inputs. An overview of state of the art in positive theory is given in the monographs [3,7]. Variety of models having positive behavior can be found in engineering, economics, social sciences, biology and medicine, etc.

Mathematical fundamentals of the fractional calculus are given in the monographs [26-28]. The positive fractional linear systems have been investigated in $[6,9,10,20]$. Stability of fractional linear continuous-time systems has been investigated in the papers $[1,11,20]$. The notion of practical stability of positive fractional linear systems has been introduced in [11]. Some recent interesting results in fractional systems theory and its applications can be found in $[2,12,29-31]$.

The minimum energy control problem for standard linear systems has been formulated and solved by J. Klamka [22-24] and for 2D linear systems with variable coefficients in [18]. The controllability and minimum energy control problem of fractional discrete-time linear systems has been investigated by Klamka in [25]. The minimum energy control of fractional positive continuous-time linear systems has been addressed in $[14,18,20]$ and for descriptor positive discrete-time linear systems in $[13,16,17,19]$.

In this paper the minimum energy control problem for positive electrical circuits will be formulated and solved. 
The paper is organized as follows. In section 2 the basic definitions and theorems of the fractional positive electrical circuits are recalled and the necessary and sufficient conditions for the reachability of the electrical circuits are given.

The main result of the paper is given in section 3 where minimum energy control problem is formulated, sufficient conditions for its solution are established and a procedure is proposed. Illustrating example of fractional positive electrical circuits is given in section 4. An extension to the method is presented in section 5. Concluding remarks are given in section 6 .

The following notation will be used: $\mathfrak{R}$ - the set of real numbers, $\Re^{n \times m}$ - the set of $n \times m$ real matrices, $\mathfrak{R}_{+}^{n \times m}$ - the set of $n \times m$ matrices with nonnegative entries and $\mathfrak{R}_{+}^{n}=\mathfrak{R}_{+}^{n \times 1}$, $M_{n}$ - the set of $n \times n$ Metzler matrices (real matrices with nonnegative off-diagonal entries), $I_{n}-$ the $n \times n$ identity matrix.

\section{Preliminaries}

The following Caputo definition of the fractional derivative will be used [20]

$$
D^{\alpha} f(t)=\frac{\mathrm{d}^{\alpha}}{\mathrm{d} t^{\alpha}} f(t)=\frac{1}{\Gamma(n-\alpha)} \int_{0}^{t} \frac{f^{(n)}}{(t-\tau)^{\alpha+1-n}} \mathrm{~d} \tau, \quad n-1<\alpha \leq n \in N=\{1,2, \ldots\},
$$

where $\alpha \in \Re$ is the order of fractional derivative and

$$
f^{(n)}(\tau)=\frac{\mathrm{d}^{n} f(\tau)}{\mathrm{d} \tau^{n}} \text { and } \Gamma(x)=\int_{0}^{\infty} e^{-t} t^{x-1} \mathrm{~d} t
$$

is the gamma function.

Consider a fractional electrical circuits composed of resistors, coils, condensators and voltage (current) sources. Using the Kirchhoff's laws we may describe the transient states in the electrical circuits by state equations $[2,4,13,20,28,29]$

$$
D^{\alpha} x(t)=A x(t)+B u(t), \quad 0<\alpha \leq 1,
$$

where $x(t) \in \mathfrak{R}^{n}, u(t) \in \mathfrak{R}^{m}$ are the state and input vectors and $A \in \mathfrak{R}^{n \times n}, \quad B \in \mathfrak{R}^{n \times m}$.

Theorem 2.1. [20] The solution of Equation (2.2) is given by

$$
x(t)=\Phi_{0}(t) x_{0}+\int_{0}^{t} \Phi(t-\tau) B u(\tau) \mathrm{d} \tau, \quad x(0)=x_{0},
$$

where

$$
\Phi_{0}(t)=E_{\alpha}\left(A t^{\alpha}\right)=\sum_{k=0}^{\infty} \frac{A^{k} t^{k \alpha}}{\Gamma(k \alpha+1)}
$$




$$
\Phi(t)=\sum_{k=0}^{\infty} \frac{A^{k} t^{(k+1) \alpha-1}}{\Gamma[(k+1) \alpha]}
$$

and $E_{\alpha}\left(A t^{\alpha}\right)$ is the Mittage-Leffler matrix function [20].

Definition 2.1. [20] The fractional system (2.2) is called the (internally) positive fractional system if and only if $x(t) \in \mathfrak{R}_{+}^{n}$ for $t \geq 0$ for any initial conditions $x_{0} \in \mathfrak{R}_{+}^{n}$ and all inputs $u(t) \in \mathfrak{R}_{+}^{m}, t \geq 0$.

Theorem 2.2. [20] The continuous-time fractional electrical circuit (2.2) is (internally) positive if and only if the matrix $A$ is a Metzler matrix and

$$
A \in M_{n}, B \in \mathfrak{R}_{+}^{n \times m} .
$$

Definition 2.2. The state $x_{f} \in \mathfrak{R}_{+}^{n}$ of the fractional electrical circuit (2.2) is called reachable in time $\left[0, t_{f}\right]$ if there exists an input $u(t) \in \mathfrak{R}_{+}^{m}, t \in\left[0, t_{f}\right]$ which steers the state of system (2.2) from zero initial state $x_{0}=0$ to the state $x_{f}$.

A real square matrix is called monomial if each its row and each its column contains only one positive entry and the remaining entries are zero.

Theorem 2.3. The positive fractional electrical circuit (2.2) is reachable in time $t \in\left[0, t_{f}\right]$ if and only if the matrix $A \in M_{n}$ is diagonal and the matrix $B \in \mathfrak{R}_{+}^{n \times n}$ is monomial.

Proof. Sufficiency. It is well known [7,20] that if $A \in M_{n}$ is diagonal then $\Phi(t) \in \mathfrak{R}_{+}^{n \times n}$ is also diagonal and if $B \in \mathfrak{R}_{+}^{n \times m}$ is monomial then $B B^{T} \in \mathfrak{R}_{+}^{n \times n}$ is also monomial. In this case the matrix

$$
R_{f}=\int_{0}^{t_{f}} \Phi(\tau) B B^{T} \Phi^{T}(\tau) \mathrm{d} \tau \in \Re_{+}^{n \times n}
$$

is also monomial and $R_{f}^{-1} \in \mathfrak{R}_{+}^{n \times n}$. The input

$$
u(t)=B^{T} \Phi^{T}\left(t_{f}-t\right) R_{f}^{-1} x_{f}
$$

steers the state of the system (2.2) from $x_{0}=0$ to $x_{f}$ since using (2.3) for $x_{0}=0$ and (2.5) we obtain

$$
\begin{aligned}
& x\left(t_{f}\right)=\int_{0}^{t_{f}} \Phi\left(t_{f}-\tau\right) B u(\tau) \mathrm{d} \tau=\int_{0}^{t_{f}} \Phi\left(t_{f}-\tau\right) B B^{T} \Phi^{T}\left(t_{f}-\tau\right) \mathrm{d} \tau R_{f}^{-1} x_{f} \\
& =\int_{0}^{t_{f}} \Phi(\tau) B B^{T} \Phi^{T}(\tau) \mathrm{d} \tau R_{f}^{-1} x_{f}=x_{f} .
\end{aligned}
$$

The proof of necessity is given in [14]. 


\section{Problem formulation and its solution}

Consider the fractional positive electrical circuit (2.2) with $A \in M_{n}$ and $B \in \mathfrak{R}_{+}^{n \times m}$ monomial. If the electrical circuit is reachable in time $t \in\left[0, t_{f}\right]$, then usually there exists many different inputs $u(t) \in \mathfrak{R}_{+}^{n}$ that steers the state of the system from $x_{0}=0$ to $x_{f} \in \mathfrak{R}_{+}^{n}$. Among these inputs we are looking for input $u(t) \in \mathfrak{R}_{+}^{n}, t \in\left[0, t_{f}\right]$ that minimizes the performance index

$$
I(u)=\int_{0}^{t_{f}} u^{T}(\tau) Q u(\tau) \mathrm{d} \tau,
$$

where $Q \in \mathfrak{R}_{+}^{n \times n}$ is a symmetric positive defined matrix and $Q^{-1} \in \mathfrak{R}_{+}^{n \times n}$.

The minimum energy control problem for the fractional positive electrical circuit (2.2) can be stated as follows.

Given the matrices $A \in M_{n}, B \in \mathfrak{R}_{+}^{n \times m}, \alpha$ and $Q \in \mathfrak{R}_{+}^{n \times n}$ of the performance matrix (3.1), $x_{f} \in \mathfrak{R}_{+}^{n}$ and $t_{f}>0$, find an input $u(t) \in \mathfrak{R}_{+}^{n}$ for $t \in\left[0, t_{f}\right]$ that steers the state vector of the system from $x_{0}=0$ to $x_{f} \in \mathfrak{R}_{+}^{n}$ and minimizes the performance index (3.1).

To solve the problem we define the matrix

$$
W\left(t_{f}\right)=\int_{0}^{t_{f}} \Phi\left(t_{f}-\tau\right) B Q^{-1} B^{T} \Phi^{T}\left(t_{f}-\tau\right) \mathrm{d} \tau,
$$

where $\Phi(t)$ is defined by (2.5). From (3.2) and Theorem 2.3 it follows that the matrix (3.2) is monomial if and only if the fractional positive electrical circuit (2.2) is reachable in time $\left[0, t_{f}\right]$. In this case we may define the input

$$
\hat{u}(t)=Q^{-1} B^{T} \Phi^{T}\left(t_{f}-t\right) W^{-1}\left(t_{f}\right) x_{f} \text { for } t \in\left[0, t_{f}\right] .
$$

Note that the input (3.3) satisfies the condition $u(t) \in \mathfrak{R}_{+}^{n}$ for $t \in\left[0, t_{f}\right]$ if

$$
Q^{-1} \in \mathfrak{R}_{+}^{n \times n} \text { and } W^{-1}\left(t_{f}\right) \in \mathfrak{R}_{+}^{n \times n} \text {. }
$$

Theorem 3.1. Let $\bar{u}(t) \in \mathfrak{R}_{+}^{n}$ for $t \in\left[0, t_{f}\right]$ be an input that steers the state of the fractional positive electrical circuit (2.2) from $x_{0}=0$ to $x_{f} \in \mathfrak{R}_{+}^{n}$. Then the input (3.3) also steers the state of the system from $x_{0}=0$ to $x_{f} \in \mathfrak{R}_{+}^{n}$ and minimizes the performance index (3.1), i.e. $I(\hat{u}) \leq I(\bar{u})$.

The minimal value of the performance index (3.1) is equal to

$$
I(\hat{u})=x_{f}^{T} W^{-1}\left(t_{f}\right) x_{f} .
$$

Proof. If the conditions (3.4) are met then the input (3.3) is well defined and $\hat{u}(t) \in \mathfrak{R}_{+}^{n}$ for $t \in\left[0, t_{f}\right]$. We shall show that the input steers the state of the system from $x_{0}=0$ to $x_{f} \in \mathfrak{R}_{+}^{n}$. Substitution of (3.3) into (2.3) for $t=t_{f}$ and $x_{0}=0$ yields 


$$
x\left(t_{f}\right)=\int_{0}^{t_{f}} \Phi\left(t_{f}-\tau\right) B \hat{u}(\tau) \mathrm{d} \tau=\int_{0}^{t_{f}} \Phi\left(t_{f}-\tau\right) B Q^{-1} B^{T} \Phi^{T}\left(t_{f}-\tau\right) \mathrm{d} \tau W_{f}^{-1} x_{f}=x_{f},
$$

since (3.2) holds. By assumption the inputs $\bar{u}(t)$ and $\hat{u}(t), t \in\left[0, t_{f}\right]$ steers the state of the system from $x_{0}=0$ to $x_{f} \in \mathfrak{R}_{+}^{n}$, i.e.

$$
x_{f}=\int_{0}^{t_{f}} \Phi\left(t_{f}-\tau\right) B \bar{u}(\tau) \mathrm{d} \tau=\int_{0}^{t_{f}} \Phi\left(t_{f}-\tau\right) B \hat{u}(\tau) \mathrm{d} \tau
$$

and

$$
\int_{0}^{t_{f}} \Phi\left(t_{f}-\tau\right) B[\bar{u}(\tau)-\hat{u}(\tau)] \mathrm{d} \tau=0 .
$$

By transposition of (3.6c) and postmultiplication by $W^{-1}\left(t_{f}\right) x_{f}$ we obtain

$$
\int_{0}^{t_{f}}[\bar{u}(\tau)-\hat{u}(\tau)]^{T} B^{T} \Phi^{T}\left(t_{f}-\tau\right) \mathrm{d} \tau W^{-1}\left(t_{f}\right) x_{f}=0 .
$$

Substitution of (3.3) into (3.7) yields

$$
\int_{0}^{t_{f}}[\bar{u}(\tau)-\hat{u}(\tau)]^{T} B^{T} \Phi^{T}\left(t_{f}-\tau\right) \mathrm{d} \tau W^{-1} x_{f}=\int_{0}^{t_{f}}[\bar{u}(\tau)-\hat{u}(\tau)]^{T} Q \hat{u}(\tau) \mathrm{d} \tau=0 .
$$

Using (3.8) it is easy to verify that

$$
\int_{0}^{t_{f}} \bar{u}(\tau)^{T} Q \bar{u}(\tau) \mathrm{d} \tau=\int_{0}^{t_{f}} \hat{u}(\tau)^{T} Q \hat{u}(\tau) \mathrm{d} \tau+\int_{0}^{t_{f}}[\bar{u}(\tau)-\hat{u}(\tau)]^{T} Q[\bar{u}(\tau)-\hat{u}(\tau)] \mathrm{d} \tau .
$$

From (3.9) it follows that $I(\hat{u})<I(\bar{u})$ since the second term in the right-hand side of the inequality is nonnegative. To find the minimal value of the performance index (3.1) we substitute (3.3) into (3.1) and we obtain

$$
I(\hat{u})=\int_{0}^{t_{f}} \hat{u}^{T}(\tau) Q u(\hat{u}) \mathrm{d} \tau=x_{f}^{T} W^{-1} \int_{0}^{t_{f}} \Phi\left(t_{f}-\tau\right) B Q^{-1} B^{T} \Phi^{T}\left(t_{f}-\tau\right) \mathrm{d} \tau W^{-1} x_{f}=x_{f}^{T} W^{-1} x_{f},
$$

since (3.2) holds.

From the above considerations we have the following procedure for computation of the optimal inputs that steers the state of the system from $x_{0}=0$ to $x_{f} \in \mathfrak{R}_{+}^{n}$ and minimizes the performance index (3.1). 
Procedure 3.1.

Step 1. Knowing $A \in M_{n}$ and using (2.5) compute $\Phi(t)$.

Step 2. Using (3.2) compute the matrix $W_{f}$ for given $A, B, Q, \alpha$ and some $t_{f}$.

Step 3. Using (3.3) compute the desired $\hat{u}(t)$ for given $x_{f} \in \mathfrak{R}_{+}^{n}$.

Step 4. Using (3.5) compute the maximal value of the performance index.

\section{Example}

Consider the fractional electrical circuit shown on Fig. 4.1 with given resistances $R_{1}, R_{2}, R_{3}$, inductances $L_{1}, L_{2}$ and source voltages $e_{1}, e_{2}$.

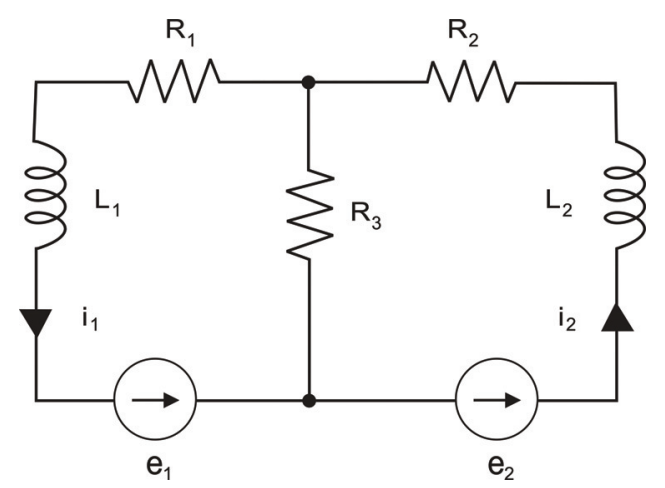

Fig. 4.1. Electrical circuit

Using the Kirchhoff's laws we can write the equations

$$
\begin{aligned}
& e_{1}=R_{3}\left(i_{1}-i_{2}\right)+R_{1} i_{1}+L_{1} \frac{\mathrm{d}^{\alpha} i_{1}}{\mathrm{~d} t^{\alpha}}, \\
& e_{2}=R_{3}\left(i_{2}-i_{1}\right)+R_{2} i_{2}+L_{2} \frac{\mathrm{d}^{\alpha} i_{2}}{\mathrm{~d} t^{\alpha}},
\end{aligned}
$$

where $0<\alpha<1$, which can be written in the form

$$
\frac{\mathrm{d}^{\alpha}}{\mathrm{d} t^{\alpha}}\left[\begin{array}{l}
i_{1} \\
i_{2}
\end{array}\right]=A\left[\begin{array}{l}
i_{1} \\
i_{2}
\end{array}\right]+B\left[\begin{array}{l}
e_{1} \\
e_{2}
\end{array}\right],
$$

where

$$
A=\left[\begin{array}{cc}
-\frac{R_{1}+R_{3}}{L_{1}} & \frac{R_{3}}{L_{1}} \\
\frac{R_{3}}{L_{2}} & -\frac{R_{2}+R_{3}}{L_{2}}
\end{array}\right], B=\left[\begin{array}{cc}
\frac{1}{L_{1}} & 0 \\
0 & \frac{1}{L_{2}}
\end{array}\right] .
$$


The fractional electrical circuit is positive since the matrix $A$ is Metzler matrix and the matrix $B$ has nonnegative entries. Note that the standard pair (4.2b) is reachable since $\operatorname{det} B \neq 0$ but it is not reachable as a positive pair.

We shall show that the fractional positive electrical circuit is reachable if $R_{3}=0$. In this case

$$
A=\left[\begin{array}{cc}
-\frac{R_{1}}{L_{1}} & 0 \\
0 & -\frac{R_{2}}{L_{2}}
\end{array}\right]
$$

and using (2.5) we obtain

$$
\Phi(t)=\sum_{k=0}^{\infty} \frac{A^{k} t^{(k+1) \alpha-1}}{\Gamma[(k+1) \alpha]}=\sum_{k=0}^{\infty}\left[\begin{array}{cc}
\left(-\frac{R_{1}}{L_{1}}\right)^{k} & 0 \\
0 & \left(-\frac{R_{2}}{L_{2}}\right)^{k}
\end{array}\right] \frac{t^{(k+1) \alpha-1}}{\Gamma[(k+1) \alpha]} .
$$

The matrix (4.4) and the matrix $B$ defined by (4.2b) are monomial and by Theorem 2.3 the fractional positive electrical circuit is reachable if $R_{3}=0$ and any values of $R_{1}, R_{2}, L_{1}, L_{2}$.

The minimum energy control problem of the fractional positive reachable electrical circuit can be stated as follows: Compute the input $\hat{u}(t) \in \mathfrak{R}_{+}^{2}$ that steers the state of the electric al circuit from zero state to $x_{f}=\left[\begin{array}{ll}1 & 1\end{array}\right]^{T}$ ( $T$ denotes the transpose) and minimizes the performance index (3.1) with

$$
Q=\left[\begin{array}{ll}
2 & 0 \\
0 & 2
\end{array}\right] .
$$

Using the procedure 3.1 we obtain the following:

Step 1. The matrix $\Phi(t)$ has the form (4.4).

Step 2. Using (3.2), (4.4), (4.2b) and (4.5) we get

$$
\begin{aligned}
W\left(t_{f}\right) & =\int_{0}^{t_{f}} \Phi\left(t_{f}-\tau\right) B Q^{-1} B^{T} \Phi^{T}\left(t_{f}-\tau\right) \mathrm{d} \tau=\int_{0}^{t_{f}} \Phi(\tau) B Q^{-1} B^{T} \Phi^{T}(\tau) \mathrm{d} \tau \\
& =\frac{1}{2} \int_{0}^{t_{f}}\left[\begin{array}{cc}
\frac{1}{L_{1}^{2}} & 0 \\
0 & \frac{1}{L_{2}^{2}}
\end{array}\right] \Phi^{2}(\tau) \mathrm{d} \tau=\left[\begin{array}{cc}
\frac{1}{2 L_{1}^{2}} & 0 \\
0 & \frac{1}{2 L_{2}^{2}}
\end{array}\right] \int_{0}^{t_{f}}\left[\sum_{k=0}^{\infty}\left[\begin{array}{cc}
-\frac{R_{1}}{L_{1}} & 0 \\
0 & -\frac{R_{2}}{L_{2}}
\end{array}\right]^{k} \frac{t^{(k+1) \alpha-1}}{\Gamma[(k+1) \alpha]}\right] \mathrm{d} \tau .
\end{aligned}
$$

Step 3. Using (3.3), (4.5) and (4.6) we obtain 


$$
\begin{aligned}
\hat{u}(t) & =Q^{-1} B^{T} \Phi^{T}\left(t_{f}-t\right) W^{-1}\left(t_{f}\right) x_{f}=\left[\begin{array}{cc}
\frac{1}{2 L_{1}} & 0 \\
0 & \frac{1}{2 L_{2}}
\end{array}\right]_{k=0}^{\infty}\left[\begin{array}{cc}
-\frac{R_{1}}{L_{1}} & 0 \\
0 & -\frac{R_{2}}{L_{2}}
\end{array}\right]^{k} \frac{t^{(k+1) \alpha-1}}{\Gamma[(k+1) \alpha]} \\
& \times\left[\begin{array}{cc}
\frac{1}{2 L_{1}^{2}} & 0 \\
0 & \frac{1}{2 L_{2}^{2}}
\end{array}\right] \int_{0}^{t_{f}}\left[\sum_{k=0}^{\infty}\left[\begin{array}{cc}
-\frac{R_{1}}{L_{1}} & 0 \\
0 & -\frac{R_{2}}{L_{2}}
\end{array}\right]^{k} \frac{t^{(k+1) \alpha-1}}{\Gamma[(k+1) \alpha]}\right] \mathrm{d} \tau\left[\begin{array}{l}
1 \\
1
\end{array}\right] .
\end{aligned}
$$

Step 4. From (3.5) and (4.6) we have the minimal value of the performance index

$$
I(\hat{u})=x_{f}^{T} W^{-1}\left(t_{f}\right) x_{f} .
$$

\section{Extension to fractional positive electrical circuits with different orders}

Consider an electrical circuit composed of resistors, $n$ capacitors and $m$ voltage (current) sources. Using the Kirchhoff's laws we may describe the transient states in the electrical circuit by the fractional differential equation

$$
\frac{\mathrm{d}^{\alpha} x(t)}{\mathrm{d} t^{\alpha}}=A x(t)+B u(t), 0<\alpha<1,
$$

where $x(t) \in \mathfrak{R}^{n}, u(t) \in \mathfrak{R}^{m}, A \in \mathfrak{R}^{n \times n}, B \in \mathfrak{R}^{n \times m}$. The components of the state vector $x(t)$ and input vector $u(t)$ are the voltages on the condensators and source voltages, respectively. Similarly, using the Kirchhoff's laws we may describe the transient states in the electrical circuit composed of resistances, inductances and voltage (current) sources by the fractional differential equation

$$
\frac{\mathrm{d}^{\beta} x(t)}{\mathrm{d} t^{\beta}}=A x(t)+B u(t), 0<\beta<1,
$$

where $x(t) \in \mathfrak{R}^{n}, u(t) \in \mathfrak{R}^{m}, B \in \mathfrak{R}^{n \times m}$. In this case the components of the state vector $x(t)$ are the currents in the coils.

Now let us consider electrical circuit composed of resistors, capacitors, coils and voltage (current) source. As the state variables (the components of the state vector $x(t)$ ) we choose the voltages on the capacitors and the currents in the coils. Using the Equations (5.1), (5.2) and Kirchhoff's laws we may write for the fractional linear circuits in the transient states the state equation 


$$
\left[\begin{array}{c}
\frac{\mathrm{d}^{\alpha} x_{C}}{\mathrm{~d} t^{\alpha}} \\
\frac{\mathrm{d}^{\beta} x_{L}}{\mathrm{~d} t^{\beta}}
\end{array}\right]=\left[\begin{array}{ll}
A_{11} & A_{12} \\
A_{21} & A_{22}
\end{array}\right]\left[\begin{array}{l}
x_{C} \\
x_{L}
\end{array}\right]+\left[\begin{array}{l}
B_{1} \\
B_{2}
\end{array}\right] u, \quad 0<\alpha, \beta<1,
$$

where the components $x_{C} \in \mathfrak{R}^{n_{1}}$ are voltages on the condensators, the components $x_{L} \in \mathfrak{R}^{n_{2}}$ are currents in the coils and the components of $u \in \Re^{m}$ are the source voltages and

$$
A_{i j} \in \mathfrak{R}^{n_{i} \times n_{j}}, B_{i} \in \mathfrak{R}^{n_{i} \times m}, \quad i, j=1,2 .
$$

Theorem 5.1. The solution of the Equation (5.3) for $0<\alpha<1 ; 0<\beta<1$ with initial conditions

$$
x_{C}(0)=x_{10} \text { and } x_{L}(0)=x_{20}
$$

has the form

$$
x(t)=\Phi_{0}(t) x_{0}+\int_{0}^{t}\left[\Phi_{1}(t-\tau) B_{10}+\Phi_{2}(t-\tau) B_{01}\right] u(\tau) \mathrm{d} \tau,
$$

where

$$
\begin{gathered}
x(t)=\left[\begin{array}{l}
x_{1}(t) \\
x_{2}(t)
\end{array}\right], x_{0}=\left[\begin{array}{l}
x_{10} \\
x_{20}
\end{array}\right], B_{10}=\left[\begin{array}{c}
B_{1} \\
0
\end{array}\right], B_{01}=\left[\begin{array}{c}
0 \\
B_{2}
\end{array}\right], \\
T_{k l}=\left\{\begin{array}{r}
{\left[\begin{array}{cc}
A_{11} & A_{12} \\
0 & 0
\end{array}\right] \text { for } k=l=0} \\
{\left[\begin{array}{cc}
0 & 0 \\
A_{21} & A_{22}
\end{array}\right] \text { for } k=0, l=1} \\
T_{10} T_{k-1, l}+T_{01} T_{k, l-1} \quad \text { for } k+l>0
\end{array}\right. \\
\Phi_{0}(t)=\sum_{k=0}^{\infty} \sum_{l=0}^{\infty} T_{k l} \frac{t^{k \alpha+l \beta}}{\Gamma(k \alpha+l \beta+1)} \\
\Phi_{1}(t)=\sum_{k=0}^{\infty} \sum_{l=0}^{\infty} T_{k l} \frac{t^{(k+1) \alpha+l \beta-1}}{\Gamma[(k+1) \alpha+l \beta]}, \\
\Phi_{2}(t)=\sum_{k=0}^{\infty} \sum_{l=0}^{\infty} T_{k l} \frac{t^{k \alpha+(l+1) \beta-1}}{\Gamma[k \alpha+(l+1) \beta]} .
\end{gathered}
$$

Proof is given in $[10,20]$. 
The extension of Theorem 5.1 to systems consisting of $n$ subsystems with different fractional orders is given in [10].

\section{Concluding remarks}

Necessary and sufficient conditions for the reachability of the fractional positive electrical circuit have been established (Theorem 2.2). The minimum energy control problem for the fractional positive electrical circuits has been formulated and solved. Sufficient conditions for the existence of a solution to the problem has been given (Theorem 3.1) and a procedure for computation of optimal input and the minimal value of performance index has been proposed. The effectiveness of the procedure has been demonstrated on the example of fractional positive electrical circuit. The presented method can be extended to positive discrete-time linear systems and to fractional positive discrete-time linear systems with bounded inputs.

\section{Acknowledgements}

This work was supported by National Science Centre in Poland under work No N N514 638940.

\section{References}

[1] Busłowicz M., Stability of linear continuous time fractional order systems with delays of the retarded type, Bull. Pol. Acad. Sci. Tech. 56(4): 319-324 (2008).

[2] Dzieliński A., Sierociuk D., Sarwas G., Ultracapacitor parameters identification based on fractional order model, Proc ECC'09, Budapest (2009).

[3] Farina L., Rinaldi S., Positive Linear Systems; Theory and Applications, J. Wiley, NY, (2000).

[4] Kaczorek T., Polynomial approach to fractional descriptor electrical circuits, Computational Models for Business and Engineering Domains - ITHEA, Rzeszow, Poland (2014).

[5] Kaczorek T., Asymptotic stability of positive fractional $2 D$ linear systems, Bull. Pol. Acad. Sci. Tech. 57(3): 289-292 (2009).

[6] Kaczorek T., Fractional positive continuous-time systems and their Reachability, Int. J. Appl. Math. Comput. Sci. 18(2): 223-228 (2008).

[7] Kaczorek T., Positive 1D and 2D systems, Springer Verlag, London (2001).

[8] Kaczorek T., Controllability and observability of linear electrical circuits, Electrical Review 87(9a): 248-254 (2011).

[9] Kaczorek T., Positivity and reachability of fractional electrical circuits, Acta Mechanica et Automatica 5(2): 42-51 (2011).

[10] Kaczorek T., Positive linear systems consisting of $n$ subsystems with different fractional orders, IEEE Trans. Circuits and Systems 58(6): 1203-1210 (2011).

[11] Kaczorek T., Practical stability of positive fractional discrete-time linear systems, Bull. Pol. Acad. Sci. Tech. 56(4): 313-317 (2008).

[12] Kaczorek T., Reachability and controllability to zero tests for standard and positive fractional discrete-time systems, Journal Européen des Systèmes Automatisés, JESA, 42(6-8): 769-787 (2008).

[13] Kaczorek T., Linear Control Systems, Research Studies Press and Wiley J., New York (1992).

[14] Kaczorek T., Minimum energy control of fractional positive continuous-time linear systems, submitted to Conf. MMAR (2013).

[15] Kaczorek T., Checking of the positivity of descriptor linear systems by the use of the shufflealgorithm, Archive of Control Sciences 21(3): 287-298 (2011). 
[16] Kaczorek T., Minimum energy control of descriptor positive discrete-time linear systems, submitted to Compel, (2013).

[17] Kaczorek T., Minimum energy control of positive discrete-time linear systems with bounded inputs, Archives of Control Sciences 23(2): 205-211 (2013).

[18] Kaczorek T., Minimum energy control of positive continuous-time linear systems with bounded inputs, Archives of Electrical Engineering 63(1): 19-27 (2014).

[19] Kaczorek T., An extension of Klamka's method of minimum energy control to fractional positive discrete-time linear systems with bounded inputs, Bull. Pol. Acad. Sci. Tech. 62(2): 227-231 (2014).

[20] Kaczorek T., Selected Problems of Fractional Systems Theory, Springer-Verlag, Berlin (2012).

[21] Kaczorek T., Klamka J., Minimum energy control of $2 D$ linear systems with variable coefficients, Int. J. of Control 44(3): 645-650 (1986).

[22] Klamka J., Controllability of Dynamical Systems, Kluwer Academic Press, Dordrecht (1991).

[23] Klamka J., Minimum energy control of 2D systems in Hilbert spaces, System Sciences 9(1-2): 33-42 (1983).

[24] Klamka J., Relative controllability and minimum energy control of linear systems with distributed delays in control, IEEE Trans. Autom. Contr. 21(4): 594-595 (1976).

[25] Klamka J., Controllability and minimum energy control problem of fractional discrete-time systems, Chapter in "New Trends in Nanotechology and Fractional Calculus", Eds. Baleanu D., Guvenc Z.B., Tenreiro Machado J.A., Springer-Verlag, New York, 503-509 (2010).

[26] Oldham K. B., Spanier J., The Fractional Calculus, Academic Press, New York (1974).

[27] Ostalczyk P., Epitome of the fractional calculus: Theory and its Applications in Automatics, Wydawnictwo Politechniki Łódzkiej, Łódź (2008) (in Polish).

[28] Podlubny I., Fractional Differential Equations, Academic Press, San Diego (1999).

[29] Radwan A.G., Soliman A.M., Elwakil A.S., Sedeek A., On the stability of linear systems with fractional-order elements, Chaos, Solitones and Fractals 40(5): 2317-2328 (2009).

[30] Solteiro Pires E.J., Tenreiro Machado J.A., Moura Oliveira P.B., Fractional dynamics in genetic algorithms, Workshop on Fractional Differenation and its Application 2: 414-419 (2006). 